\title{
Accurate Distance Estimation for VANET Using Nanointegrated Devices
}

\author{
Ashraf Nasr ${ }^{1,2}$, Samir A. Elsagheer Mohamed ${ }^{2,3}$ \\ ${ }^{1}$ Radiation Engineering Department, NCRRT, Cairo, Egypt \\ ${ }^{2}$ College of Computer, Qassim University, Buryadah, KSA \\ ${ }^{3}$ Electrical Engineering Department, Faculty of Engineering, South Valley University, Aswan, Egypt \\ Email: Ashraf.nasr@gmail.com, samirahmed@yahoo.com
}

Received March 29, 2012; revised April 28, 2012; accepted May 9, 2012

\begin{abstract}
Vehicular Ad-Hoc Networks (VANET) is a research venue that promises for many useful applications. Most of these applications require a precise real-time positioning system for each vehicle. However, practically the existing techniques are still not accurate and hence not suitable for some critical applications. In this paper, we will focus on the most critical ones which are the collision avoidance, and collision warning, or lane-tracking. Collision occurs when the distance between nearby vehicles decreases rapidly. Hence, an accurate and precise knowledge of the distance among each vehicle and all the surrounding vehicles has to be obtained to enable a realistic collision avoidance service. We propose to use the carbon nanotube network (CNT) integrated with other nano-devices that can provide accuracy in the order of millimeters. In this paper, theoretical investigations and mathematical formulations are presented. The obtained results show the effectiveness and accuracy of the proposed methodology.
\end{abstract}

Keywords: Vehicular Ad-Hoc Networks; Positioning Systems; Wireless Networks; Nanotechnology Devices; CNT Sensors

\section{Introduction}

Recently, there are many research efforts on a new technology named as Vehicular Ad-Hoc Network (VANET) [1-5]. In VANET, vehicles will be equipped by wireless transceiver that allows each vehicle to communicate with the surrounding vehicles on the street. This is referred to vehicle-to-vehicle $(\mathrm{V} 2 \mathrm{~V})$ communication. In addition, the roads will be reengineering by installing wireless equipments on the sides of roads. These equipments are referred as the Road-Side Units (RSUs). The RSUs are connected together using wired infrastructure or in some cases using the Wireless Mesh Networks (WMS) infrastructure. The RSUs are connected to the central servers. In this case, the vehicles can communicate also with the RSUs as they move. This type of communication is referred as Vehicle-to-Infrastructure (V2I) communication. Both V2V and V2I communications form moving ad-hoc networks. Using this network architecture, the vehicles can be connected all the time with the infrastructure network and with the surrounding vehicles. Many useful applications can be implemented with VANET. Some promising applications may include collision avoidance, cooperative driving, automatic driving, navigation and probe vehicle data that increase vehicular safety and reduce traffic congestion, and offer access to the internet and entertainment applications. Another kind of services that could not be realistic without VANET includes the autonomous street lightening system [6], smart traffic light management system [7], and automatic fracture recording and monitoring system [8].

On the other hand, VANET technology is still in the research phase, where there are some challenges to be undertaken before the real deployment of VANET technology on the streets. The most critical one is the positioning system (aka localization system) for the vehicles. In other words, this system answers the question "How to provide each vehicle by its real time location?" There exist several positioning techniques that are suitable for many applications [9-18]. Each application or service provided by VANET has its own requirement of the positioning system. Some applications may tolerate some error level in the positioning information. Other applications require a very accurate and reliable positioning system. Example of the first category is the traffic management applications. Most of the safety applications on the other hand require the second category. Some examples are the collision avoidance, automatic driving and lane tracking. The accuracy of positioning system must be within the centimeter. In addition, its availability must be guaranteed. If it fails for some time, catastrophic circumstances may occur such as collisions or car crashes. 
The most famous positioning system is the GPS (by using a set of satellites that feeds information about the position of a GPS receiver). However, all the existing positioning techniques including the GPS have several drawbacks. Lack of accuracy of the resulting measurements is the most unacceptable disadvantage. For example, GPS devices can produce an error of up to 50 meters [10]. This accuracy may seem to be acceptable for several applications. On the other side, as mentioned before, other applications like collision avoidance, automatic driving and lane tracking demand precise and accurate positioning information. Therefore, the existing positioning techniques including GPS are not suitable for this kind of applications in VANET. Recently an enhancement to the GPS referred as the Differential GPS (DGPS) consisting in installing expensive ground stations can improve the accuracy significantly. However, GPS and DGPS and the similar techniques do not work in tunnels, undergrounds, and in high dense building areas, because the signal cannot be received or received very weak.

On the other hand, nanotechnology is a field of science that is concerned by controlling matter on a scale between $1-100 \mathrm{~nm}$. It provides solutions for sensing, actuation, radio, embedding intelligence into the environment, power efficient computing memory, energy sources, human-machine interaction, materials, mechanics, manufacturing and environment issues [19-26].

Nanotechnology wireless ad-hoc networks with large number of extremely low cost, low power nodes are studied. For example, all required components of a wireless sensor node, i.e., a sensing unit, a processing unit, and a power unit have already been demonstrated with nanoelments, such as carbon nanotubes (CNs) [27-32]. Nanotechnology transceivers devices have important role in the network applications. It increases the sensorial of each vehicle with other neighboring things. We, thus, will provide a theoretical study for obtaining accurate inter-vehicle distance measures based on the CNT networks.

In this paper, we propose an accurate inter-vehicle distance estimation model that can be used for accurate short distance estimation. The range can be less than 20 meters and with accuracy that can be within several millimeters. The model is referred as the Vehicular Carbon Nanotube (VCNT) networks. It is clear that this system can be used in the critical applications like the collision avoidance. The system is accurate and reliable and can work in all areas. If this system is used in conjunction with DGPS, it could provide a full range of distance and position estimation in real time.

The rest of this paper is organized as follows: In Section 2, the related works and research efforts are given. Theoretical model of vehicle carbon nanotube network is denoted in Section 3. Finally, the conclusions and the future works are given in Section 4.

\section{Related Works}

There exits two categories of positioning systems: indoors techniques $[33,34]$, which are not suitable for VANET due to its cost and the limited distance they support. Another research works in VANET positioning includes the following techniques. First, the signalstrength-based techniques, where the receiver calculates an estimate of its location based on the received signal strength from several wireless access points. These techniques are not accurate. Reported results based on this technique shows poor accuracy $[1,13]$ (around 50 meters). An interesting work that proposes a positioning system based on the Received Signal Strength (RSS) for VANET is proposed in [35]. The system uses the same equipments of VANET to reduce the cost. By solving the channel interference and the near-far problems, the system gives in practice an accuracy of maximum absolute error of 2 meters.

Second, Time-Of-Arrival (TOA)-Based techniques, which are based on the travelled distance from the base station to the receiver of a known signal. This is the solution adopted by GPS [19]. As we mentioned before, its practical measurements are not acceptable for VANET, as it lacks the accuracy. In addition, the Signal cannot be received in some places (tunnels, undergrounds, and near dense areas of building). TOA techniques require a perfect synchronization between the clocks of the base stations and the receivers. This cannot be guaranteed except by using atomic clocks which are very expensive. Otherwise the accuracy is compromised. Third, techniques which are based on the round trip time [19]. The receiver sends a small packet to the base station and wait for a reply from it. The elapsed time is proportional to the distance between it and the base station. By some calculations the receiver can know its location provided that it can talk to at least three base stations each one knows its accurate location. Again the distance between the base stations and the receiver must be large to obtain good results.

From nanotechnology point of view, the significant interesting application is wireless ad-hoc networks with large number of extremely low-cost, low power elements. For example all the required components of a wireless sensor node, i.e., a sensing unit, a processing unit, a transceiver unit, and a power unit have already been demonstrated with nanoelments, such as carbon nanotubes $(\mathrm{CN})$ [25]. Hence, the focus in this paper is on $\mathrm{CN}$ integrated system. The physical operation of a nanotube antenna is significantly different from that of a classical antenna for wireless communication. When it acts as receiver, the nanotube absorbs electromagnetic waves and converts them to mechanical vibrations. Since the nano- 
tube is charged, these vibrations cause changes in the electric field at the cathode right across the nanotube and the induced current is processed to recover the incident signal. Since the mechanism of the nanotube ante na is electromechanical, the nanoreceiver has to overcome the acoustic noise, which is effective directly on the nanotube, as well as the thermal noise, effective at the rear end of the antenna [34]. In another case, when it acts as transmitter, the previous procedure is reversed, i.e., the nanotube is mechanically oscillating at the frequency of the carrier signal. By applying a dc voltage to the nanotube, charge is concentrated at the tip of the nanotube. Therefore, when the nanotube oscillates it radiates an oscillating electromagnetic field [35].

\section{Model of Vehicle Carbon Nanotube Network}

A nanosensor is not necessarily a device merely reduced in size to a few nanometers, but a device that makes use of the unique properties of nanomaterials and nanoparticles to detect and measure new types of events in the nanoscale [26]. Recent advancements in molecular and carbon electronics such as Carbon Nanotube $(\mathrm{CN})$ have opened the door to a new generation of electronic nanocomponents such as nanobatteries, nano-memories, logical circuitry in the nanoscale and even nano-antennas [28]. CN based sensors have shown many benefits over their past counterparts and are suitable candidates in this nanotechnology driven age [29]. As one can see in Figure 1, the integrated carbon nanotube network for vehicle, (VCNT), denotes a concept of operation (See also Figure 2). It depends essentially on the $\mathrm{CN}$ and MicroElectro-Mechanical Systems (MEMS) based sensor nodes. Terahertz range; medium infrared region, (1 - 10 $\mathrm{THz}$ ) is received by nano-sensors while megahertz range is received by MEMs sensor and nano-transeciver. The megahertz wave can be used to remote control the nanosensor system by classical transmitters in macro-range [26]. After that the received signal will be amplified and processed by the nano-stages as depicted. Nano-batteries like quantum dot solar cell can be used to supply all stages of nano-devices. To achieve communications between nodes in VCNT, the nodes must be activated, either externally by a centralized controller, or internally in a sequential manner as the activation signal propagates in the network in a multihop fashion.

The activation signal is an electromagnetic wave has certain duration, tuned according to the resonance frequency of the nanotube antennas of the nodes to be activated [35]. Really, the activation of adjacent nodes changes with time and vehicle's position. This requires a software to be processed by the nano-processor and loaded into the memory in Figure 1.

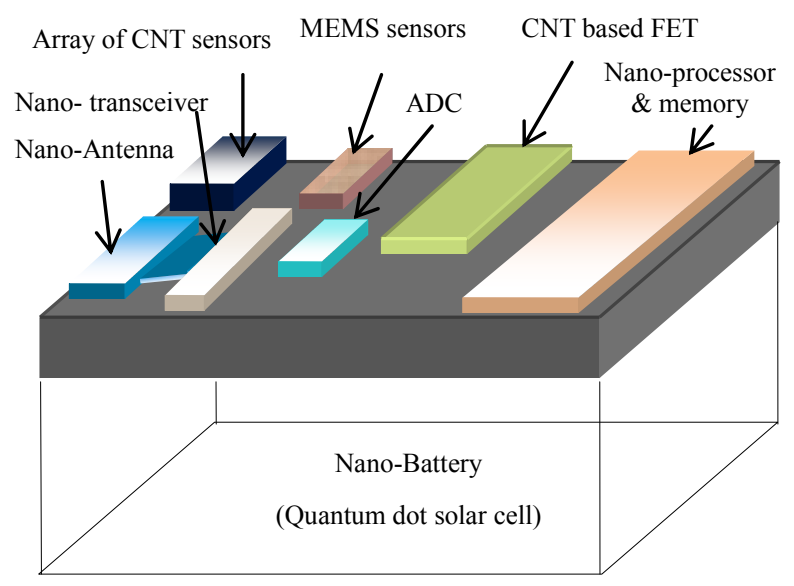

Figure 1. Integrated carbon nanotube network for vehicle (VCNT).

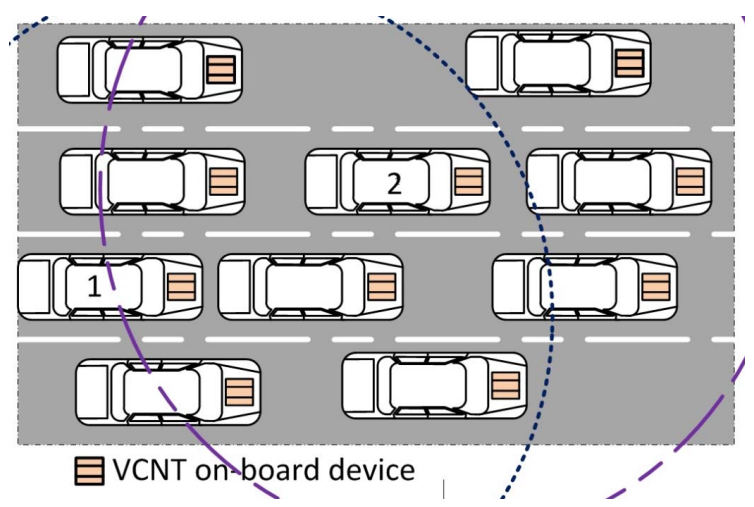

Figure 2. Vehicular Carbon Nanotube Networks (VCNT). Each circle is a VCNT, virtually having a vehicle on the center (ex. Vehicle \#1 and \#2 in the Figure). VCNT is formed in an ad-hoc maner. VCNT on-board device measures the distance between the vehicle and all the surrounding vehicles in that VCNT.

Here, description of the construction of VCNT and mathematical model will be demonstrated for the overall capacity and the capacity per meter between nodes. CNT have a period of one decade and each year new applications are proposed [30]. We propose this type of sensors as a better candidate for inter-vehicular distance estimation. As described above, the accuracy of the existing distance estimation techniques are not suitable for some VANET applications. VCNT can be a good choice to solve this issue, by providing cheep accurate inter-vehicle distance estimation in the short range (within 20 meter). In CNT operation; the variation of conductance current is depending on the detection of physical, or chemical, or biological effects. Because of the scale of CNT is down in size, an enhancement of network performance will be achieved. Higher capacity or larger bandwidth is accomplished with increasing oscillation frequency. CNT frequency is $1 / L$ more than traditional wireless communication; where $L$ is the linear scale dimension [31]. Here, 
VCNT model is proposed to cover the smaller distance between a vehicle and surrounding ones. Each vehicle establishes one or more carbon nanotube node network as shown in Figure 2. Thus, VCNT operation is similar to the Mobile Ad-hoc Networks (MANET); where nodes (vehicles) join and leave the network at any time. In addition, due to the variance of the vehicles' speed, the vehicles form many dynamic VCNTs as they move.

The total VCNT is the sum of vehicles in the zone at specific time. The number of nodes (vehicles) is changed according to the place and time in ad-hoc manner. So, we will start by determining the internal link capacity between nodes. The capacity per meter of a channel from specified transmitter to receiver is given by Shannon's famous formula [32]:

$$
C_{T R}=\{B W \ln [1+S / N] / d\}
$$

where, $B W$ is the bandwidth of the communication, $S / N$ is the signal-to-noise ratio (SNR) of the link, and $d$ is the distance. The previous formula states the relation between the theoretical of clean maximum bit rate of clean data (or low bit error rate) with a given average signal power that can be sent through an FM analog communication channel subject to additive, white, and Gaussiandistribution noise interference. Figure 3 depicts the channel capacity with distance at different values of SNR. According to the distance between adjacent vehicles, the strength of the transmitted power is used for the accurate determination of the surrounding empty distance.

Also, the obtained channel capacity for CNT is higher than the traditional wireless network as it depends on the $B W$ which is in the range of $\mathrm{THz}$ in our case. In VCNT model, assume that each point source is omni-directionally radiation and a network of the randomly distributed $n$ nodes is spread over a circular area, A. Each connection between the adjacent nodes (vehicles) has a determined channel capacity, $C_{T R}$. Also, there is guard distance $\Delta$, which ensure channel transmission do not overlap [32]. Then the maximum wireless capacity in the case of VCNT can be given by:

$$
C_{\max }=\sqrt{8 / \pi} C T R / \Delta \sqrt{n} \sum_{k=1}^{n} \sqrt{A} / K
$$

where, $\sqrt{A} / K$ is the expected nearest-neighbor distance, the summation denotes the total distance that data must travel.

Figure 4 shows the relation between the maximum capacity and radius of circular radiation source at different values of SNR. It is evident to consider the superiorrity of VCNT capacity over traditional wireless networks at smaller distance as notices in Figure 4. One can notice that the total capacity is increased with circular radius as a result of the number of accessing nodes is increased. From the theoretical phase, it is due to the summation in Equation (1), when VCNT includes more nodes, the $C_{\max }$

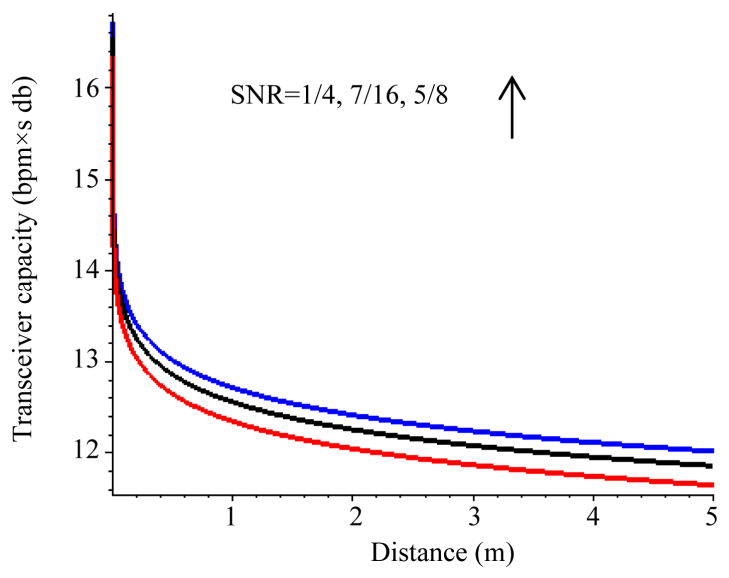

Figure 3. Transmitter-receiver capacity between two vehicles as a function of distance at different values of SNR. Lower capacity is noticeable at longer distance. Also, more efficient link capacity can be achieved at higher values of SNR.

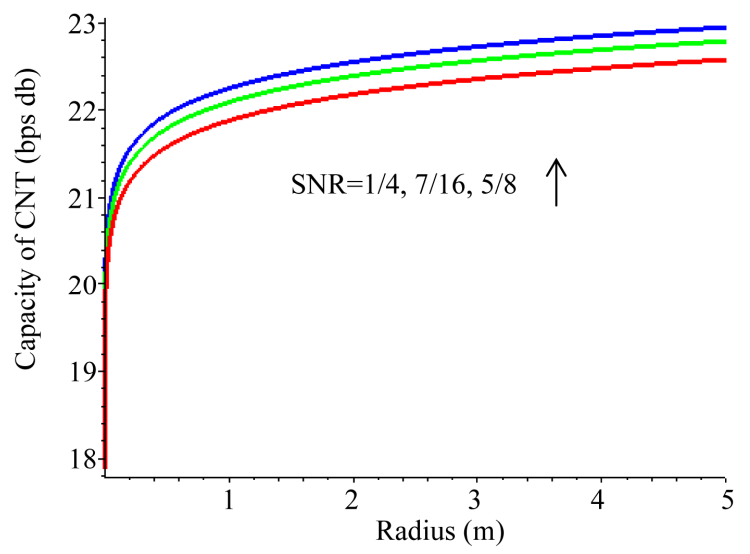

Figure 4. The maximum capacity of carbon nanotube network (CNT) with omni-directionally circular source radius at different values of SNR. For nanotube distance; $d=10 \mathrm{~m}$, $B W=10 \mathrm{THz}$.

will be dramatically greater than before. To have a wide view about the behavior of VCNT, Figures 5 and $\mathbf{6}$ depict the concept of dependency of maximum capacity into each of radiation radius; $r$ and the distance between nanotube transmitter and receiver; $d$, at two values of bandwidth. One can recognize that as the distance increased, Cmax will decrease as discussed before in Figure 3. Also, when $B W$ takes higher values the data rate will increase which impact dramatically upon the total maximum capacity.

From the above equations, the distance can be estimated from SNR and capacity (see Figures 5 and 6). Finally, it is desirable to obtain accurate distance estimation at small space between vehicles and continuously cover suitable area surrounding it at any instant of time while it moves. This will direct us to denote more attention for studying and analyzing the important role of 


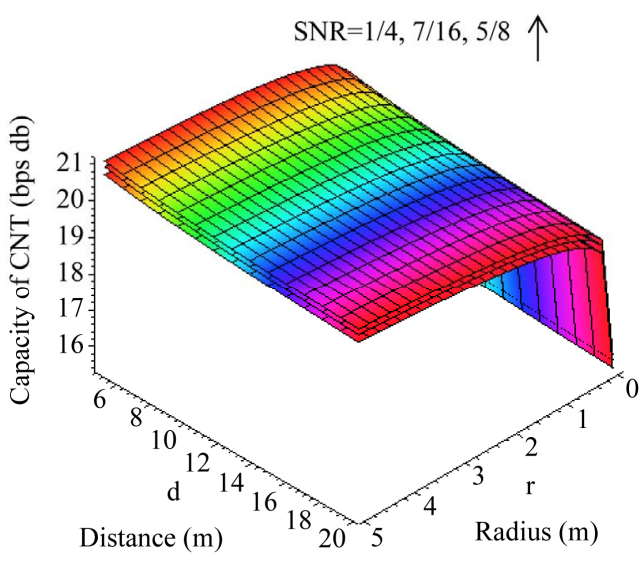

Figure 5. The maximum capacity of carbon nanotube network (CNT) with omni-directionally circular source radius and nanotube distance at different values of SNR when $B W$ $=0.1 \mathrm{THz}$.

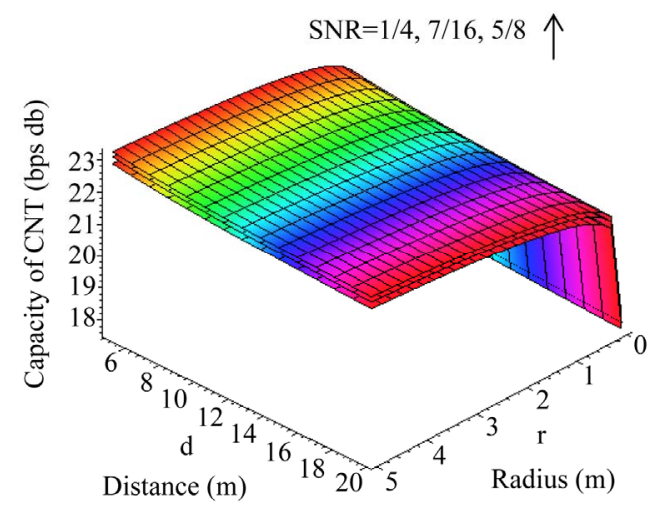

Figure 6. The maximum capacity of carbon nanotube network (CNT) with omni-directionally circular source radius and nanotube distance at different values of SNR when $B W$ $=10$ THz.

nano-processor in the future for activating or deactivating the surrounding nodes at any time and at any speed of vehicle.

\section{Conclusions and Future Directions}

In this paper, an integrated nanotechnology model that can be used to precisely cover and assign the locations in near distance positions is presented. Moreover, we aimed to obtain distance estimation within several meters with an accuracy of several millimeters.

Additionally, the theoretical model for Vehicular Nano Tube Networks (VCNT) is presented. The aim of this theoretical phase is to complete the picture that the nanotechnology system for vehicular positioning can be exploited to give more accuracy and high performance with lower cost and power consumption. Future research direction may include the validation of the model in realistic environment.

\section{REFERENCES}

[1] H. Hartenstein and K. P. Laberteaux, "A Tutorial Survey on Vehicular Ad-Hoc Networks," IEEE Communications Magazine, Vol. 46, No. 6, 2008, pp. 164-171. doi:10.1109/MCOM.2008.4539481

[2] J. Harri, F. Filali and C. Bonnet, "Mobility Models for Vehicular Ad-Hoc Networks: A Survey and Taxonomy," IEEE Communications Surveys \& Tutorials, Vol. 11, No. 4, 2009, pp. 19-41.

[3] R. Tatchikou and S. Biswas, "Vehicle-to-Vehicle Packet forwarding Protocols for Cooperative Collision Avoidance," Proceedings of the Global Telecommunications Conference, Saint Louis, 28 November-2 December 2005, 5 pages.

[4] A. Nandan, S. Das, G. Pau, M. Y. Sanadidi and M. Gerla, "Cooperative Downloading in Vehicular Ad Hoc Wireless Networks," Proceedings of Wireless On-Demand Network Systems and Services, Saint Moritz, January 2005, pp. 32-41.

[5] S. Kato, S. Tsugawa, K. Tokuda, T. Matsui and H. Fujii, "Vehicle Control Algorithms for Cooperative Driving with Automated Vehicles and Intervehicle Communications," IEEE Transactions on Intelligent Transportation Systems, Vol. 3, No. 3, 2002, pp. 155-161. doi:10.1109/TITS.2002.802929

[6] S. A. Mohamed, "Smart Street Lighting Control and Monitoring System for Electrical Power Saving Using VANET," Ad Hoc Networks Journal, Elsevier, 2012.

[7] S. A. Mohamed, "Intelligent Traffic Management System for the Future VANET Technology," under Submission.

[8] S. A. Mohamed, A. Nasr and G. A. Ansari, "Precise Positioning Systems for Vehicular Ad-Hoc Networks," International Journal of Wireless \& Mobile Networks, Vol. 4, No. 2, 2012, pp. 215-265.

[9] M. Z. Rahman and L. Kleeman, "Paired Measurement Localization: A Robust Approach for Wireless Localization," IEEE Transactions on Mobile Computing, Vol. 8, No. 8, 2009, pp. 1087-1102. doi:10.1109/TMC.2008.173

[10] K. Yu and Y. J. Guo, "Anchor Global Position Accuracy Enhancement Based on Data Fusion," IEEE Transactions on Vehicular Technology, Vol. 58, No. 3, 2009, pp. 16161623. doi:10.1109/TVT.2008.928636

[11] R. Singh, M. Guainazzo and C. S. Regazzoni, "Location Determination Using WLAN in Conjunction with GPS Network," Proceedings of the Vehicular Technology Conference (VTC-Spring), Genova, 2004 IEEE 59th, Vol. 5, DIBE, pp. 2695-2699.

[12] R. W. Ouyang, A. K.-S. Wong and C.-T. Lea, "Received Signal Strength-Based Wireless Localization via Semidefinite Programming: Noncooperative and Cooperative Schemes," IEEE Transactions on Vehicular Technology, Vol. 59, No. 3, 2010, pp. 1307-1318. doi:10.1109/TVT.2010.2040096

[13] B.-C. Liu and K.-H. Lin, "SSSD-Based Mobile Positioning: On the Accuracy Improvement Issues in Distance and Location Estimations," IEEE Transactions on Vehicular Technology, Vol. 58, No. 3, 2009, pp. 1245-1254.

[14] B.-C. Liu, K.-H. Lin and J.-C. Wu, "Analysis of Hyper- 
bolic and Circular Positioning Algorithms Using Stationary Signal-Strength-Difference Measurements in Wireless Communications," IEEE Transactions on Vehicular Technology, Vol. 55, No. 2, 2006, pp. 499-509.

[15] K. K. Mada, H.-C. Wu and S. S. Iyengar, "Efficient and Robust EM Algorithm for Multiple Wideband Source Localization," IEEE Transactions on Vehicular Technology, Vol. 58, No. 6, 2009, pp. 3071-3075. doi:10.1109/TVT.2009.2012495

[16] R. Parker and S. Valaee, "Vehicular Node Localization Using Received-Signal-Strength Indicator," IEEE Transactions on Vehicular Technology, Vol. 56, No. 6, 2007, pp. 3371-3380.

[17] L. Wischof, A. Ebner and H. Rohling, "Information Dissemination in Self-Organizing Intervehicle Networks," IEEE Transactions on Intelligent Transportation Systems, Vol. 6, No. 1, 2005, pp. 90-101. doi:10.1109/TITS.2004.842407

[18] C. Komar and C. Ersoy, "Location Tracking and Location Based Service Using IEEE 802.11 WLAN Infrastructure," Proceedings of the European Wireless Workshop, 24-27 February 2004.

[19] M. Porretta, P. Nepa, G. Manara and F. Gianne, "Location, Location, Location," IEEE Vehicular Technology Magazine, Vol. 3, No. 2, 2008, pp. 20-29. doi:10.1109/MVT.2008.923969

[20] A. Nasr and M. B. El_Mashade,"Theoretical Comparison between Quantum-Well and Dot Infrared Photodetectors," IEEE Proceedings of Optoelectronic, Vol. 153, No. 4, 2006, pp. 183-190.

[21] A. Nasr, "Performance of Quantum Wire Infrared Photodetectors under Illumination Conditions," Optics \& Laser Technology, Vol. 41, No. 7, 2009, pp. 871-876. doi:10.1016/j.optlastec.2009.03.001

[22] A. Nasr, A. Aboshosha and S. M. Al-Adl, "Dark Current Characteristics of Quantum Wire Infrared Photodetectors" IET Optoelectronics, Vol. 1, No. 3, 2007, pp. 140145. doi:10.1049/iet-opt:20060089

[23] A. Nasr, "Spectral Responsivity of the Quantum Wire Infrared Photodetectors," Optics \& Laser Technology, Vol. 41, No. 3, 2009, pp. 345-350. doi:10.1016/j.optlastec.2008.05.020

[24] A. Nasr, "Detectivity Performance of Quantum Wire Infrared Photodetectors," Journal of Optical Communications, Vol. 32, No. 2, 2011, pp. 101-106.
[25] V. Ermolov, M. Heino, A. Kärkkäinen, R. Lehtiniemi, N. Nefedov, P. Pasanen, Z. Radivojevic, M. Rouvala and T. Ryhänen "Significance of Nanotechnology for Future Wireless Devices and Communications," 18th Annual IEEE International Symposium on Personal, Indoor and Mobile Radio Communications, Athens, 2007.

[26] I. F. Akyildiz and J. M. Jornet, "Electromagnetic Wireless Nanosensor Networks," Nano Communication Networks, Vol. 1, No. 1, 2010, pp. 3-19.

[27] J. P. M. She and J. T. W. Yeow, "Nanotechnology-Enabled Wireless Sensor Networks: Overcoming the Limitations from a Device Perspective," Proceedings of the IEEE International Conference on Mechatronics and Automation, Canada, 2005, Vol. 3, pp. 1610-1617.

[28] P. Burke, C. Rutherglen and Z. Yu, "Nanotubes and Nanowires," World Scientific, Vol. 44, 2007, 136 pages. doi:10.1142/9789812708274

[29] M. Z. Atashbar, C. Baratto, G. Faglia and G. Sberveglieri, "Functionalized Single Wall Carbon Nanotubes Based Gas Sensor," 5th IEEE Conference on Sensors, Daegu, 22-25 October 2006, pp. 247-250.

[30] R. Pathak, S. Joshi, P. Parandkar and S. Katiyal, "Modeling and Reliability Analysis of CNT and MEMS Based Wireless Sensor Network," Journal of Mathematics and Technology, Vol. 1, No. 1, 2010, pp. 136-146.

[31] E. L. Wolf, "Nanophysics and Nanotechnology," 2nd Edition, Wiley-VCH, Hoboken, 2004.

[32] S. F. Bush and S. Goel, "Graph Spectra of Carbon Nanotube Networks," 1st International Conference on Nano-Networks and Workshops, Lausanne, 14-16 September 2006, pp. 1-10.

[33] A. K. M. Hossain, H. N. Van, Y. Jin and W.-S. Soh, "Indoor Localization Using Multiple Wireless Technologies," IEEE Internatonal Conference on Mobile Adhoc and Sensor Systems, Pisa, 8-11 October 2007, pp. 1-8.

[34] S. Misra, G.-L. Xue and S. Bhardwaj, "Secure and Robust Localization in a Wireless Ad Hoc Environment," IEEE Transactions on Vehicular Technology, Vol. 58, No. 3, 2009, pp. 1480-1489. doi:10.1109/TVT.2008.926616

[35] S. A. Mohamed, "Why the Accuracy of the Received Signal Strengths as a Positioning Technique Was Not Accurate?" International Journal of Wireless \& Mobile Networks, Vol. 3, No. 3, 2011, pp. 69-82. doi:10.5121/ijwmn.2011.3306 